\title{
Partitions and Edge Colourings of Multigraphs
}

\author{
Alexandr V. Kostochka* and Michael Stiebitz ${ }^{\dagger}$ \\ Submitted: May 23, 2007; Accepted: Jul 1, 2008; Published: Jul 6, 2008
}

\begin{abstract}
Erdős and Lovász conjectured in 1968 that for every graph $G$ with $\chi(G)>\omega(G)$ and any two integers $s, t \geq 2$ with $s+t=\chi(G)+1$, there is a partition $(S, T)$ of the vertex set $V(G)$ such that $\chi(G[S]) \geq s$ and $\chi(G[T]) \geq t$. Except for a few cases, this conjecture is still unsolved. In this note we prove the conjecture for line graphs of multigraphs.
\end{abstract}

\section{Introduction}

It was conjectured by Erdős and Lovász (see Problem 5.12 in [2]) that for every graph $G$ with $\chi(G)>\omega(G)$ and any two integers $s, t \geq 2$ with $s+t=\chi(G)+1$, there is a partition $(S, T)$ of the vertex set $V(G)$ such that $\chi(G[S]) \geq s$ and $\chi(G[T]) \geq t$. The only settled cases of this conjecture that we know are $(s, t) \in\{(2,2),(2,3),(2,4),(3,3),(3,4),(3,5)\}$ (see $[1,3,5,6])$. In this note we prove for the line graphs of multigraphs the following slightly stronger statement.

Theorem 1 Let $s$ and $t$ be arbitrary integers with $2 \leq s \leq t$. If the line graph $L(G)$ of some multigraph $G$ has chromatic number $s+t-1>\omega(L(G))$, then it contains a clique $Q$ of size s such that $\chi(L(G)-Q) \geq t$.

It will be convenient to prove the theorem in the language of edge colorings of multigraphs. Every multigraph in this note is finite, undirected and has no loops.

The edge set and the vertex set of $G$ is denoted by $V(G)$ and $E(G)$ respectively. For a vertex $v$ of $G$, the degree, $d(v)$, of $v$ in $G$ is the number of edges incident with $v$. The set $N_{v}$ of all neighbours of $v$ in $G$ may have much smaller size than $d(v)$.

${ }^{*}$ Department of Mathematics, University of Illinois, Urbana, IL 61801 and Institute of Mathematics, Novosibirsk 630090, Russia. E-mail address: kostochk@math.uiuc.edu. This material is based upon work supported by NSF Grants DMS-0400498 and DMS-06-50784 and grant 06-01-00694 of the Russian Foundation for Basic Research.

${ }^{\dagger}$ Institute of Mathematics, Technische Universität Ilmenau, D-98684 Ilmenau, Germany. E-mail address: Michael.Stiebitz@tu-ilmenau.de. 
The chromatic index of $G$, denoted by $\chi^{\prime}(G)$, is the chromatic number of its line graph $L(G)$; in other words, it is the smallest number of colours with which the edges of $G$ may be coloured so that no two adjacent edges receive the same colour.

A triangle in $G$ is a set of three mutually adjacent vertices in $G$, and the edges of a triangle are those edges in $E(G)$ joining the vertices of the triangle. The maximum number of edges in a triangle in $G$ will be denoted by $\tau(G)$. Furthermore, let $\Delta(G)$ denote the maximum degree of $G$, and let $\omega^{\prime}(G)=\max \{\tau(G), \Delta(G)\}$. Clearly, $\omega^{\prime}(G)$ is the clique number of the line graph of $G$ and hence $\chi^{\prime}(G) \geq \omega^{\prime}(G)$.

\section{Proof of Theorem 1}

For given $2 \leq s \leq t$, suppose that $G$ is a counterexample with the fewest vertices. Then $G$ is connected. Since $\chi^{\prime}(G)>\omega^{\prime}(G) \geq \tau(G), G$ contains at least four vertices. By Shannon's theorem [4], $\chi^{\prime}(G) \leq\left\lfloor\frac{3}{2} \Delta(G)\right\rfloor$. Consequently, $s \leq \Delta(G)$.

By an s-star of $G$ we mean a pair $\left(E^{\prime}, v\right)$ such that $E^{\prime} \subseteq E(G)$ is a set of $s$ edges incident with the vertex $v$. For an $s$-star $\left(E^{\prime}, v\right)$, let $X\left(E^{\prime}, v\right)$ denote the set of all vertices of $G$ joined by an edge of $E^{\prime}$ with $v$.

Let $\left(E^{\prime}, v\right)$ be an arbitrary $s$-star of $G$. The set $E^{\prime}$ forms an $s$-clique in $L(G)$. Since $G$ is a counterexample to our theorem, we have $\chi^{\prime}\left(G-E^{\prime}\right) \leq t-1$. Let $G^{\prime}=G-E^{\prime}$, and let $\varphi: E\left(G^{\prime}\right) \longrightarrow\{1, \ldots, t-1\}$ be a $(t-1)$-edge-colouring of $G^{\prime}$. For each vertex $x$ of $G$, let

$$
\varphi(x)=\left\{\varphi(e) \mid e \in E\left(G^{\prime}\right) \text { is incident with } x\right\} \text { and } \bar{\varphi}(x)=\{1, \ldots, t-1\} \backslash \varphi(x) .
$$

Since $s+t-1=\chi^{\prime}(G)>\omega^{\prime}(G) \geq \Delta(G)$ and all $s$ edges of $E^{\prime}$ are incident with $v$, the degree of $v$ in $G^{\prime}=G-E^{\prime}$ is at most $t-2$ and, therefore,

(a) $\bar{\varphi}(v) \neq \emptyset$.

Next, we claim that

(b) for every colour $\alpha \in \bar{\varphi}(v)$ and for any two distinct vertices $x, y \in X\left(E^{\prime}, v\right)$, there is an edge $e \in E\left(G^{\prime}\right)$ joining $x$ and $y$ with $\varphi(e)=\alpha$. Consequently, $\left|X\left(E^{\prime}, v\right)\right| \leq 2$.

Proof. Suppose to the contrary that no edge joining $x$ and $y$ is colored with $\alpha$. For $u \in\{x, y\}$, there is an edge $e_{u} \in E^{\prime}$ joining $u$ and $v$. Colour the $s-1$ edges of $E^{\prime} \backslash\left\{e_{x}\right\}$ with colours $t, t+1, \ldots, t+s-2$, so that $e_{y}$ is coloured with $t$. If $\alpha \in \bar{\varphi}(x)$, we can colour the edge $e_{x}$ with $\alpha$. Otherwise, there is an edge $e \in E(G) \backslash E^{\prime}$ incident with $x$ colored with $\alpha$. Since $e$ is not incident with $y$, we can recolour $e$ with colour $t$ and then colour $e_{x}$ with $\alpha$. In both cases we obtain a $(t+s-2)$-edge-colouring of $G$, a contradiction to $s+t-1=\chi^{\prime}(G)$.

(c) Let $w$ be a vertex of $G$ with $d(w) \geq s$. Then, for the neighbourhood $N_{w}$ of $w$ in $G$, we have $\left|N_{w}\right| \geq 2$, and any two vertices of $N_{w}$ are adjacent in $G$. Furthermore, if $s \geq 3$, then $\left|N_{w}\right|=2$. 
Proof. If $N_{w}$ consists only of a single vertex $w^{\prime}$, then $d\left(w^{\prime}\right) \geq d(w) \geq s$. Since $G$ is connected and has at least four vertices, $w^{\prime}$ has a neighbour $x \neq w$. Hence there is an $s$-star $\left(E^{\prime}, w^{\prime}\right)$ of $G$ with $w, x \in X\left(E^{\prime}, w^{\prime}\right)$. From (a) and (b) it then follows that $x$ and $w$ are adjacent in $G$, a contradiction to $\left|N_{w}\right|=1$. This proves that $\left|N_{w}\right| \geq 2$. If $x, y$ are two distinct neighbours of $w$, then there is an $s$-star $\left(E^{\prime}, w\right)$ with $x, y \in X\left(E^{\prime}, w\right)$. Then (a) and (b) imply that $x$ and $y$ are adjacent. If $s \geq 3$ and $\left|N_{w}\right| \geq 3$, then there is an $s$-star $\left(E^{\prime}, w\right)$ such that $\left|X\left(E^{\prime}, w\right)\right| \geq 3$, a contradiction to (b). Hence (c) is proved.

To complete the proof of Theorem 1, we consider two cases.

Case 1: $s \geq 3$. Since $s \leq \Delta(G)$, there is a vertex $u$ in $G$ with $d(u) \geq s$. By (c), $N_{u}$ consists of two vertices, say $x$ and $y$, and these two vertices are adjacent in $G$. Since $G$ is a connected graph with at least four vertices, either $N_{x}$ or $N_{y}$ contains more than two vertices, say $\left|N_{x}\right| \geq 3$. Then (c) implies that $d(x)<s$. Let $E_{1}$ denote the set of all edges of $G$ joining $x$ with $u$ or $y$. Furthermore, let $E_{2}$ denote the set of all edges of $G$ joining $u$ with $y$. Then $2 \leq\left|E_{1}\right|<s$ and $\left|E_{1}\right|+\left|E_{2}\right| \geq s$. Hence, there is a nonempty subset $E_{2}^{\prime}$ of $E_{2}$ such that $E^{\prime}=E_{1} \cup E_{2}^{\prime}$ contains exactly $s$ edges. Since $E^{\prime}$ is an $s$-clique in $L(G)$, by the choice of $G$, we have $\chi^{\prime}\left(G-E^{\prime}\right) \leq t-1$. Let $G^{\prime}=G-E^{\prime}$, and let $\varphi: E\left(G^{\prime}\right) \longrightarrow\{1, \ldots, t-1\}$ be any $(t-1)$-edge-colouring of $G^{\prime}$. If $\varphi(u)=\{1, \ldots, t-1\}$, then $\{u, x, y\}$ is a triangle with at least $s+t-1$ edges, a contradiction to $\tau(G)<\chi^{\prime}(G)=s+t-1$. Hence there is a colour $\alpha \in \bar{\varphi}(u)$. Choose two edges $e_{1} \in E_{1}$ and $e_{2} \in E_{2}^{\prime}$. Colour the $s-1$ edges of $E^{\prime} \backslash\left\{e_{1}\right\}$ with colours $t, t+1, \ldots, t+s-2$ so that $e_{2}$ is coloured with $t$. If $\alpha \in \bar{\varphi}(x)$, then we can colour the edge $e_{1}$ with $\alpha$. Otherwise, there is an edge $e \in E(G) \backslash E^{\prime}$ such that $e$ is incident with $x$ and $\varphi(e)=\alpha$. Since all edges joining $x$ with $y$ are in $E^{\prime}$, the edge $e$ is not incident with $y$ and we can recolour $e$ with $t$ and then colour $e_{1}$ with $\alpha$. In both cases we obtain a $(t+s-2)$-edge colouring of $G$, a contradiction to $s+t-1=\chi^{\prime}(G)$.

Case 2: $s=2$. Since $s \leq \Delta(G)$, it follows from (c) that $G$ contains a triangle $T=$ $\{x, y, z\}$.

For $u \in\{y, z\}$, there is an edge $e_{u}$ in $G$ joining $u$ and $x$. The pair $\left(E^{\prime}, x\right)$ with $E^{\prime}=\left\{e_{y}, e_{z}\right\}$ is an $s$-star of $G$ and, therefore, $\chi^{\prime}\left(G-E^{\prime}\right) \leq t-1$. Let $G^{\prime}=G-E^{\prime}$, and let $\varphi: E\left(G^{\prime}\right) \longrightarrow\{1, \ldots, t-1\}$ be any $(t-1)$-edge-colouring of $G^{\prime}$.

Since $T$ contains at most $\tau(G) \leq \chi^{\prime}(G)-1=t$ edges and two of these edges are not coloured, some colour $\alpha \in\{1, \ldots, t-1\}$ is not present on edges of $T$. By (b), $\alpha \in \varphi(x)$. Hence the following two subcases finish the proof of the theorem.

Case 2.1: $\alpha \in \bar{\varphi}(y) \cup \bar{\varphi}(z)$. By the symmetry between $y$ and $z$, we can suppose that $\alpha \in \bar{\varphi}(y)$. By (a) and (b), there is a colour $\beta \in \bar{\varphi}(x)$ and an edge $e^{\prime}$ of colour $\beta$ joining $y$ and $z$. Uncolour $e^{\prime}$ and colour $e_{z}$ with $\beta$. This results in a $(t-1)$-edge-colouring $\varphi^{\prime}$ of $G-E^{\prime \prime}$, where $E^{\prime \prime}=\left\{e_{y}, e^{\prime}\right\}$. Then $\alpha \in \bar{\varphi}^{\prime}(y)$ and no edge joining $x$ and $z$ has colour $\alpha$. Since $\left(E^{\prime \prime}, y\right)$ is an $s$-star of $G$, this is a contradiction to (b).

Case 2.2: $\alpha \in \varphi(x) \cap \varphi(y) \cap \varphi(z)$. This means that for every $u \in T$, there is an edge $e^{u} \in E\left(G^{\prime}\right)$ of colour $\alpha$ joining $u$ and some vertex $v_{u} \notin T$. Let $\beta \in \bar{\varphi}(x)$ and $P$ be the component containing $x$ of the subgraph $H_{\alpha, \beta}$ induced by the set of edges $\left\{e \in E\left(G^{\prime}\right) \mid \varphi(e) \in\{\alpha, \beta\}\right\}$. Obviously, $P$ is a path starting at $x$. By (b), there is an edge $e^{\prime}$ of colour $\beta$ joining $y$ and $z$ and we eventually consider two cases. 
Subcase A: Edge $e^{\prime}$ does not belong to $P$. If we interchange the colours $\alpha$ and $\beta$ on $P$, then we obtain a new $(t-1)$-edge-colouring $\varphi^{\prime}$ of $G^{\prime}$. Then $\varphi^{\prime}$ is a $(t-1)$-edge-colouring of $G^{\prime}$ with $\alpha \in \bar{\varphi}^{\prime}(x)$ and $\varphi^{\prime}\left(e^{y}\right)=\varphi^{\prime}\left(e^{z}\right)=\alpha$. In particular, no edge of $G^{\prime}=G-E^{\prime}$ joining $y$ and $z$ has colour $\alpha$, a contradiction to (b).

Subcase B: Edge $e^{\prime}$ belongs to $P$. In this case, $e^{y}$ and $e^{z}$ also belong to $P$. By symmetry, we may assume that the subpath $P^{\prime}$ of $P$ joining $y$ with $x$ does not contain $z$. Uncolour $e^{\prime}$ and colour $e_{y} \in E^{\prime}$ with $\beta$. This results in a $(t-1)$-edge-colouring $\varphi^{\prime}$ of $G-\left\{e_{z}, e^{\prime}\right\}$ for which Subcase A with $z$ in place of $x$ and $e_{y}$ in place of $e^{\prime}$ holds. Since Subcase A is settled, this finishes the whole proof.

\section{References}

[1] W. G. Brown and H. A. Jung, On odd circuits in chromatic graphs, Acta Math. Acad. Sci. Hungar. 20 (1999), 129-134.

[2] T. R. Jensen and B. Toft, Graph Coloring Problems, Wiley Interscience, New York, 1995.

[3] N. N. Mozhan, On doubly critical graphs with chromatic number five, Technical Report 14, Omsk Institute of Technology, 1986 (in Russian).

[4] C. E. Shannon, A theorem on coloring the lines of a network, J Math. Phys. 28 (1949), $148-151$.

[5] M. Stiebitz, $K_{5}$ is the only double-critical 5-chromatic graph, Discrete Math. 64 (1987), 91-93.

[6] M. Stiebitz, On $k$-critical $n$-chromatic graphs. In: Colloquia Mathematica Soc. János Bolyai 52, Combinatorics, Eger (Hungary), 1987, 509-514. 